\title{
Fallopian Tube Anastomotic Leakage
}

National Cancer Institute

\section{Source}

National Cancer Institute. Fallopian Tube Anastomotic Leakage. NCI Thesaurus. Code C78292.

Leakage due to breakdown of a fallopian tube anastomosis. 